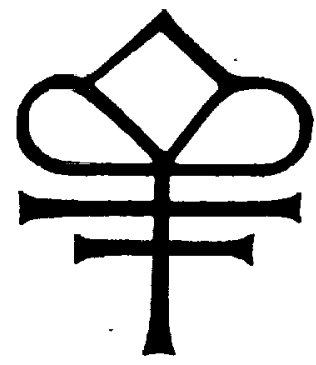

\title{
N93-15590
}

\section{A MONTE CARLO ANALYSIS OF THE LIQUID XENON TPC AS GAMMA-RAY TELESCOPE}

E. Aprile, A. Bolotnikov, D. Chen and R. Mukherjee

Department of Physics and

Columbia Astrophysics Laboratory, Columbia University

538 West 120th Street, New York, NY 10027, USA

Presented at the:

"International Conference on Liquid Radiation Detectors"

April $\tau-10,1992$, Waseda University, Tokyo, Japan

To appear in:

Nuclear Instruments and Methods in Physics Research A, 1992

\section{COLUMBIA UNIVERSITY}

DEPARTMENTS OF

\section{PHYSICS and ASTRONOMY \\ NEW YORK, NEW YORK 10027}

Columbia Astrophysics Laboratory Contribution Number 493 


\title{
A MONTE CARLO ANALYSIS OF THE LIQUID XENON TPC AS GAMMA-RAY TELESCOPE
}

\author{
E. Aprile, A. Bolotnikov, D. Chen and R. Mukherjee \\ Physics Department and \\ Columbia Astrophysics Laboratory \\ Columbia University, New York, NY 10027
}

\section{ABSTRACT}

Extensive Monte Carlo modeling of a coded aperture $\gamma$-ray telescope based on a high resolution liquid xenon TPC has been performed. Results on efficiency, background reduction capability and source flux sensitivity are presented. We discuss in particular the development of a reconstruction algorithm for events with multiple interaction points. From the energy and spatial information, the kinematics cr Compton scattering is used to identify and reduce background events, as well as to improve the detector response in the few $\mathrm{MeV}$ region. Assuning a spatial resolution of $1 \mathrm{~mm}$ RMS and an energy resolution of $4.5 \%$ FWHM at $1 \mathrm{MeV}$, the algorithm is capable of reducing by an order of magnitude the background rate expected at balloon altitude, thus significantly improving the telescope sensitivity.

\section{INTRODUCTION}

Significant progress in experimental $\boldsymbol{\gamma}$-ray astrophysics can only be achieved with the development of instruments with improved imaging capability. Efforts to improve the sensitivity of a $\gamma$-ray telescope without a corresponding improvement in angular resolution will result in source confusion in the field-of-view (FOV) and will limit the mapping of extended source distributions. Two important scientific objectives which typify the need for detectors with improved imaging capability, along with good sensitivity and energy resolution, are the $1.8 \mathrm{MeV}$ line emission from radioactive ${ }^{26} \mathrm{Al}$ and the $0.511 \mathrm{MeV}$ positron-electron annihilation emission. For both cases a precise study of the spatial distribution of the emission will uniquely identify the sources of radiation. Among the proposed novel techniques for irnaging astrophysical $\gamma$-ray sources in the low to medium energy region, the liquid xenon Time Projection Chamber (LXe-TPC) is recognized as very promising and worth a strong R\&D effort. Like an electronic bubble chamber, a TPC is capable of visualizing the complex histories of $\gamma$-ray events with multiple interactions, initiated by either Compton scattering or pair production. As a result of this imaging, efficient background rejection is also achieved, reducing the requirement for a masaive anti-coincidence shield of the type that is required for germanium and sodium iodide detectors. From the energy and spatial information available for each point of interaction, Compton kinematics enables the reconstruction of the direction of the incoming $\gamma$-ray. The direction is not unique unless the Compton scattered electron is also imaged. Given the short range of low energy electrons in liquid xenon, this is not possible for a practical spatial resolution of the order of millimeters. In the LXe-TPC Compton telescope, as proposed by us in [1], the angular resolution for the $\mathrm{MeV}$ region is limited by the small separation between two successive $\gamma$-ray interactions.

To overcome this limitation and release the strong requirement on the TPC spatial resolution, we propose to combine the imaging capability of the LXe-TPC with that of the coded aperture technique, for studying astrophysical sources in the few MeV region with arcminute accuracy. In a coded aperture telescope, a mask consisting of a twodimensional array of opaque and transparent elements is placed between the source and a position sensitive $\gamma$-ray detector. Every source within the FOV casts a shadow of part of the mask onto the detector. By properly decoding the pattern obtained at the detector plane, an image of the source is inferred. The successful application of coded aperture imaging in the low energy $\gamma$-ray region has been demonstrated by two balloon borne telescopes : the New Hampshire "Directional Gamma-Ray Telescope", DGT [2] and the Caltech "Gamma-Ray Imaging Payload", GRIP [3], and more recently by the SIGMA telescope aboard the GRANAT satellite [4]. All these telescopes use scintillators as position sensitive $\gamma$-ray detectors. The advantage of the LXe-TPC/coded telescope is in its unique capability to suppress background over a wide energy range, thus improving the sensitivity to weak sources, while maintaining an excellent detection efficiency, energy and angular resolution.

In this paper the technical aspects of the LXe-TPC development are not considered. For this we refer to previous publications by Aprile et al. [5]. We present here initial results from a Monte Carlo analysis of the expected performance of the LXe-TPC coupled to a coded aperture as $\gamma$-ray telescope for $0.3-10 \mathrm{MeV}$.

\section{MONTE CARLO ANALYSIS AND RESULTS}

\subsection{Telescope Model}

The model of the $\gamma$-ray telescope used in the Monte Carlo simulation is schematically shown in Fig.1. It consists of a coded aperture mask, located 1 meter above the LXe-TPC $\gamma$-ray detector. An active CsI shield has been included. In practice, the background rejection capability 
of the TPC reduces the need for massive anti-coincidence shield. The sensitive area of the TPC is $39 \times 28 \mathrm{~cm}^{2}$. The total depth of liquid xenon is $10 \mathrm{~cm}$. The detector's dimensions and mask-detector separation have been determined on the basis of the existing gondola for the DGT telescope that we plan to use for the first balloon flight of the telescope [6]. The TPC is operated in the ionization mode. Both the energy and the spatial distribution of each ionizing event within its sensitive volume is measured. The coordinates in the X-Y plane are inferred from the signals induced on two orthogonal wire planes, while the coordinate along the Z-direction is inferred from the drift time measured with respect to a zero time provided by the fast scintillation signal. As indicated by our first experimental results on the induction signals in a 3.5 liter LXe-TPC, to be published elsewhere, the spatial resolution in the plane is better than $S / \sqrt{12}$ where $S$ is the spacing between the induction wires. For the spatial resolution on the coordinate along the drift axis we have previously measured $\sigma_{2}=0.18 \mathrm{~mm}$ [5]. For the Monte Carlo calculations, a resolution of $\sigma_{x}=\sigma_{y}=1 \mathrm{~mm}$ and $\sigma_{z}=0.2 \mathrm{~mm}$ has been assumed, along with the experimental energy resolution of $4.5 \%$ FWHM at $1 \mathrm{MeV}$, extrapolated according to a $E^{-1 / 2}$ law [7].

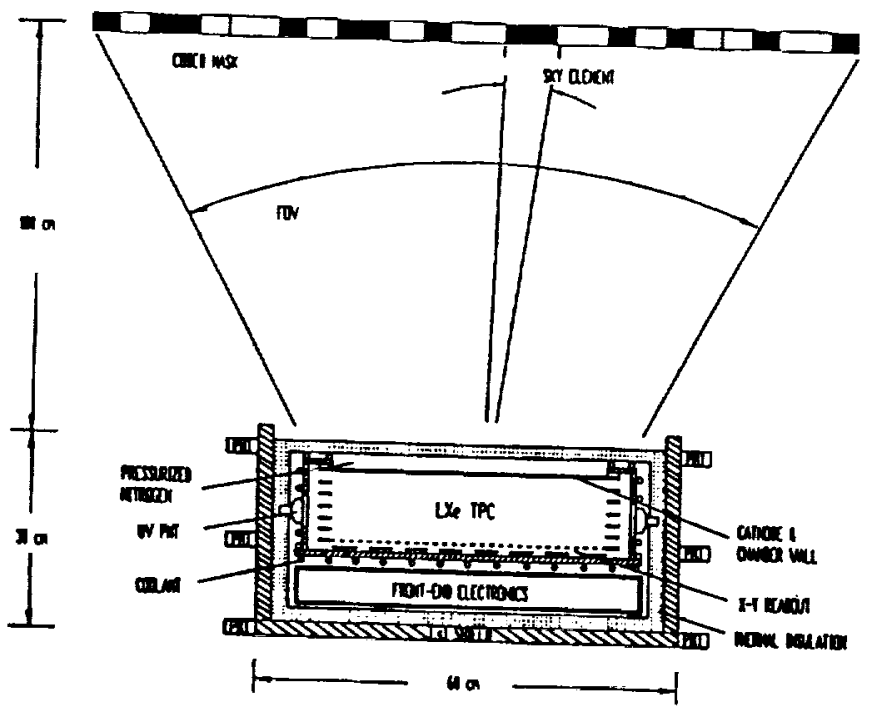

Fig. 1. Schematic of the LX $\sim$ TPC/coded mask $\gamma$-ray telescope.

The mask is a $2 \times 2$ mosaic of a basic Uniformly Redundant Array (URA) [8]. It consists of a $43 \times 41$ element pattern of $0.91 \mathrm{~cm} \times 0.68 \mathrm{~cm} \times 1.2 \mathrm{~cm}$ thick tungsten blocks. With the assumed separation of 1 meter between mask and the detector, an angular pixel size of $0.65^{\circ} \times$ $0.49^{\circ}$ and a FOV of $28^{\circ} \times 20^{\circ}$ is defined. Much finer angular resolution is obtained by increasing the mask-detector separation. The precision in point source localization depends, however, also on the detector's spatial resolution and the statistical significance of the source data. We have estimated that a precision at the arcminute level is possible with a practical spatial resolution of 1 or 2 num.
2.2 Reconstruction Algorithm based on Compton Kinematics

The interaction of $\gamma$-rays in liquid xenon is dominated by Compton scattering and pair production, for energies larger than fow hundred $\mathrm{keV}$. The typical event registered by the TPC is therefore characterized by multiple interaction points before the original $\gamma$-ray energy is fully deposited in the liquid. This is illustrated in Fig. 2 where the distribution of the number of interaction points for three different $\gamma$-ray energies is plotted. The probability that the
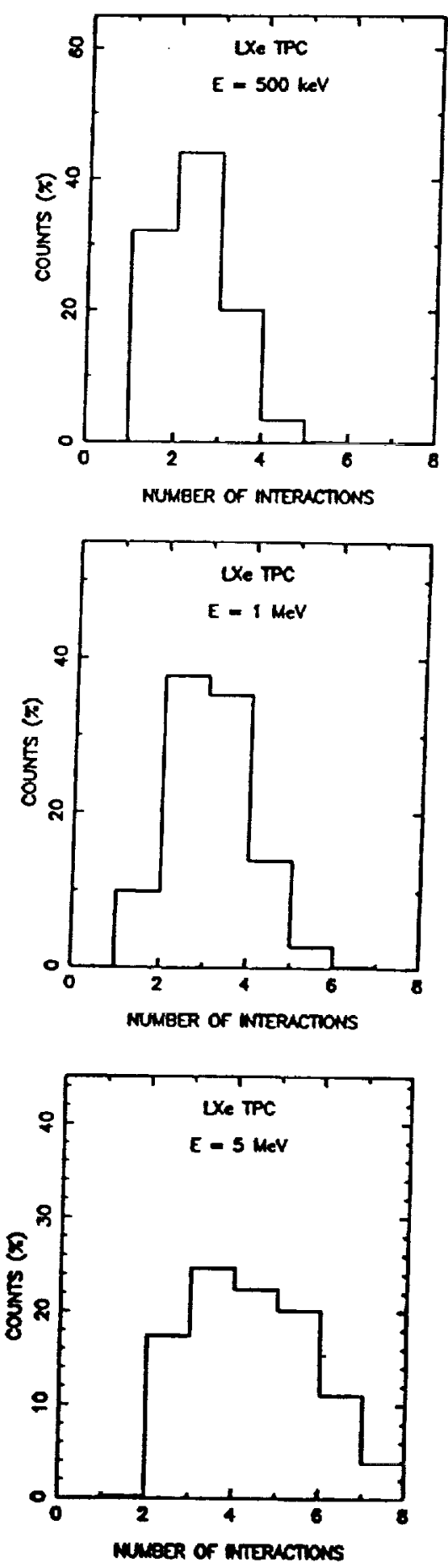

Fis. 2. Distribution of interaction points for $\gamma$-ray energies of $500 \mathrm{keV}, 1 \mathrm{MeV}$ and $5 \mathrm{MeV}$. 
first interaction point is a Compton scattering is $90 \%$ at $1 \mathrm{MeV}$, decreasing to about $25 \%$ at $10 \mathrm{MeV}$. To improve the signal/noise level in the few $\mathrm{MeV}$ region where multiple Compton events dominate, an algorithm based on the kinematics of the Compton process was developed and tested. The algorithm uses the capability of the LXe-TPC to visualize each incoming $\gamma$-ray event and to reconstruct the initial direction from the energy deposited in each interaction point and the coordinates of the points. Consider an event in which a $\gamma$-ray produces $\mathrm{N}$ energy deposition points. Let $W_{0}$ be the energy of the incoming photon, $W_{i}$ the energy of the scaltered photon, $E_{i}$ the energy of the scattered electron and $\cos \theta_{i}$ the cosine of the scattering angle. If the coordinates and the energy depositions for each interaction point, as well as the right order of the successive interactions are known, then from energy and momentum conservation we can write for each interaction point:

$$
1-\cos \theta_{i}=\frac{1}{W_{i}}-\frac{1}{W_{i-1}}
$$

where $W_{i}=W_{i+1}+E_{i+1}$, for $i=1, N-1$. For any given distribution of interaction points generated inside the sensitive volume, we now assume that the last interaction point is a photo-absorption and that the initial direction of the incoming $\gamma$-ray is known. Calling

$$
\frac{E_{i}}{1-\cos \theta_{i}}=\alpha_{i}
$$

(1) becomes

$$
W_{i-1} \cdot W_{i}=\alpha_{i}
$$

Similarly, for the $(i+1)^{\text {th }}$ point,

$$
W_{i} \cdot W_{i+1}=\alpha_{i+1}
$$

From (3) and (4),

$$
W_{i}=\frac{\alpha_{i}-\alpha_{i+1}}{E_{i}+E_{i+1}}
$$

Also, if the total energy is contained, $W_{0}$ is

$$
W_{0}=\frac{\alpha_{1}}{E_{2}+E_{3}+\cdots E_{N}}
$$

Alternatively, from energy conservation alone we have an independent estimate of the energy of the scattered photon at each point. We express this as $W_{i}^{\prime}$.

$$
W_{i}^{\prime}=E_{i+1}+\cdots E_{N}
$$

for $i=0, N-1$. To test the validity of the assumption that the total energy is contained, as well as if the assumed initial direction of the $\gamma$-ray is kinematically possible, the function

$$
f=\sum_{i=0}^{N-1}\left(W_{i}-W_{i}^{\prime}\right)^{2}
$$

is minimized for all possible combination of points. The calculation takes into account the errors on the energy and spatial coordinates.

\subsection{Detection Efficiency}

The Monte Carlo calculations were based on the EGS4 computer code [9]. The excellent gamma-ray detection efficiency of liquid xenon over a wide energy range is shown in Fig.3. The full energy peak efficiency was calculated for gamma-rays in the range $0.3-10 \mathrm{MeV}$, normally incident on the forward face of a $10 \mathrm{~cm}$ deep liquid xenon detector. The energy of the events in the sample was required to be totally contained. The use of a coded mask

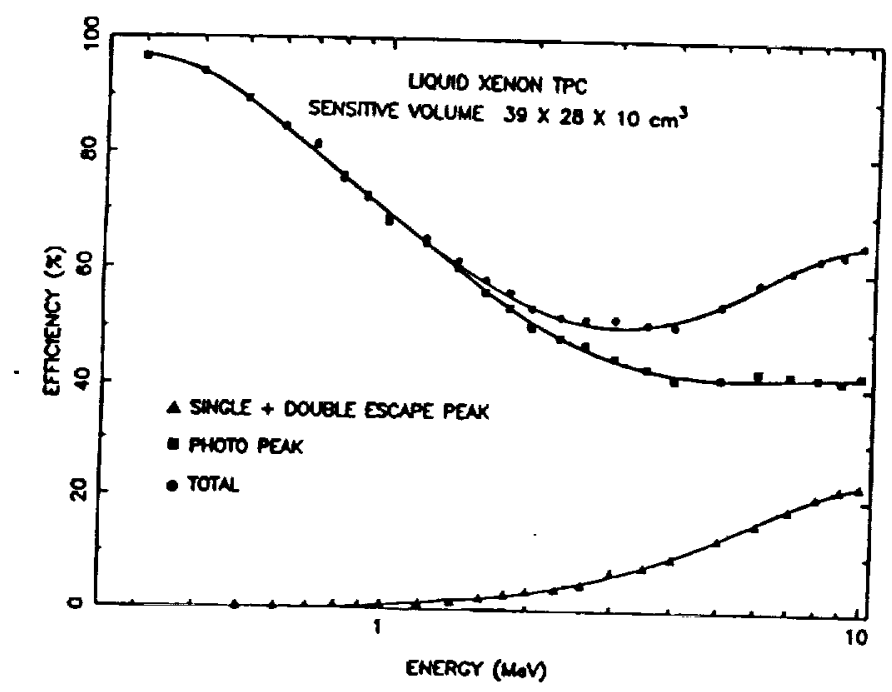

Fig. 3. Efficiency of the LXe-TPC for normally incident photons.

reduces the detection efficiency by a factor of two, assuming 100\% opacity of the closed mask elements. This is shown in the upper curve of Fig. 4. The effect of $3 \mathrm{~mm}$ stainless steel detector wall was also accounted for. The effect of the Compton reconstruction algorithm, described in section 2.2, on the telescope efficiency is also shown in Fig. 4 with the points $\square$. Because the algorithm is tajlored for events which start with a Compton scattering, all events with a single photo-absorption or which start with a pair production are rejected. To recover the detection

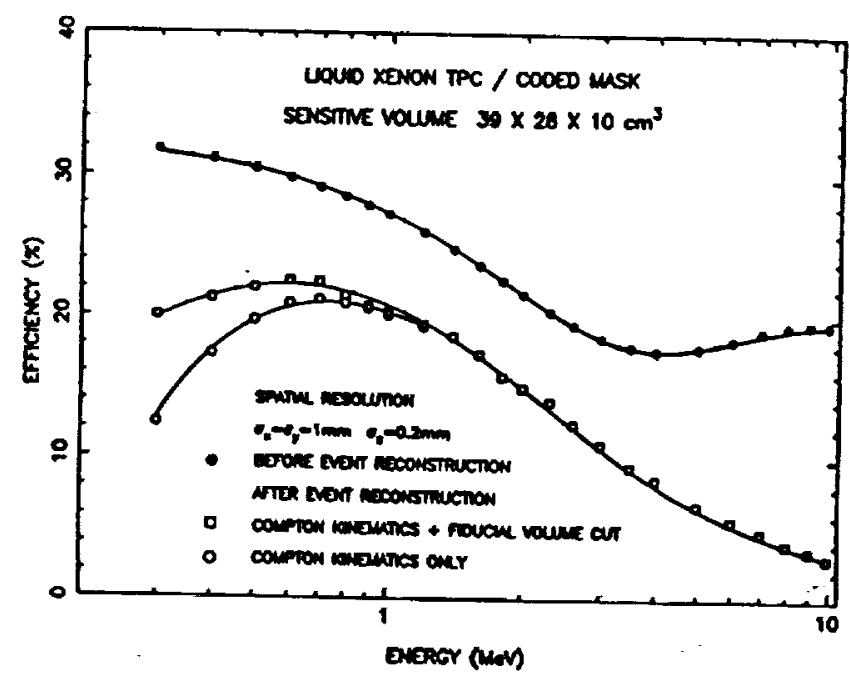

Fig. 4. Bficiency of the IXa-TPC/coded mask, before and after event reconstruction. 
efficiency at low and high energy, different reconstruction techniques can be applied. For example, the majority of low energy photons coming from the source will interact in the top region of the LXe-TPC. Fiducial volume cuts for single site events can therefore drastically improve the sig$\mathrm{nal} /$ noise ratio. This is shown in Fig. 4 with the points $\bullet$. All single-site events reconstructed outside the first $1 \mathrm{~cm}$ layer of liquid xenon from the top were rejected. For high energy events which start with a pair production, tracking of the electron/positron can be used, on an event-by-event basis, to estinate the $\gamma$-ray direction from the opening angle of the pair. Such an algorithm has however not been applied as one can see from the drastic drop in efficiency at energies above $\sim 2 \mathrm{MeV}$.

The capability of the reconstruction algorithm to reject events under the Compton continuum is shown in Fig. 5 , which shows the ratio of the total counts under the peak to that under the Compton continuum. A large improvement in this ratio is achieved for the $\mathrm{MeV}$ region. The excellent capability of the same algorithm to recognize $\gamma$ ray events entering the TPC normally from the bottom was also tested. Results are shown in Fig. 6. For this calculation total energy containment was not required.

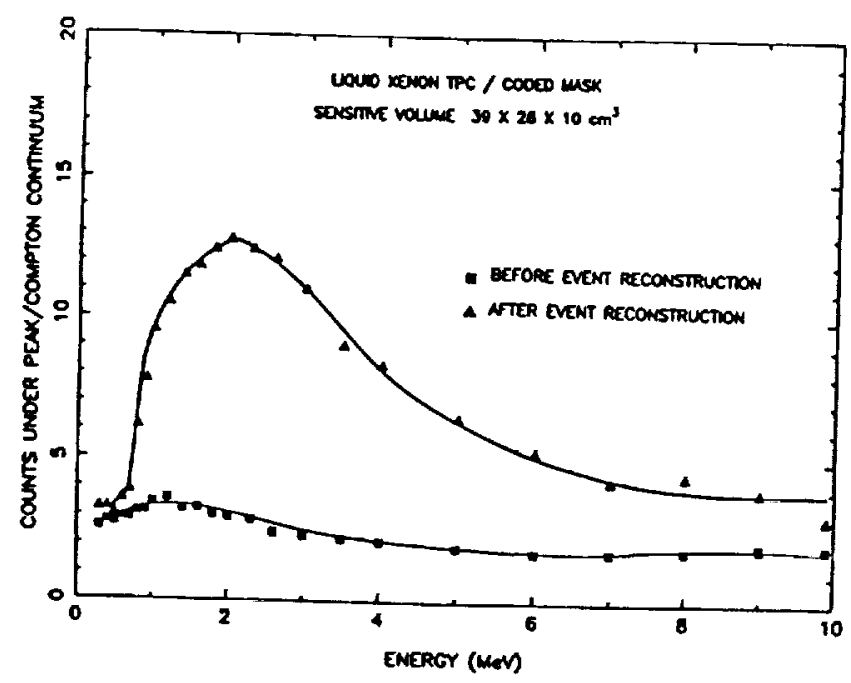

Fig. 5. Ratio of events under the peak to that under the Compton continuum for the LXe-TPC/coded mask before and after event reconstruction.

\subsection{Background Reduction and Flux Sensitivity.}

The ultimate flux sensitivity is perhaps the most relevant "figure-of-merit" of a $\gamma$-ray telescope. Among the factors which determine the sensitivity are: (i) the energy of the $\gamma$-rays and (ii) the detector's effective area, energy and spatial resolution, the exposure time and the total background rate. To calculate the background expected in the LXe-TPC/coded mask telescope at balloon altitudes, we have taken into account the dominant atmospheric and cosmic diffuse components, entering the forwand aperture of the telescope or leaking through a thick $(5 \mathrm{~cm})$ CsI active shield. The flux and angular distribution of the atmospheric $\gamma$-rays used in the calculation were taken from the parameterized forms given by Costa et al. [10] and the cosmic diffuse spectrum used was that given in refer- ence [11]. The internal backgrounds from natural radioactivity, cosmic ray induced radioactivity and activation of instrument materials has been neglected, as we expect to reject the majority of these single site events. Pulse shape

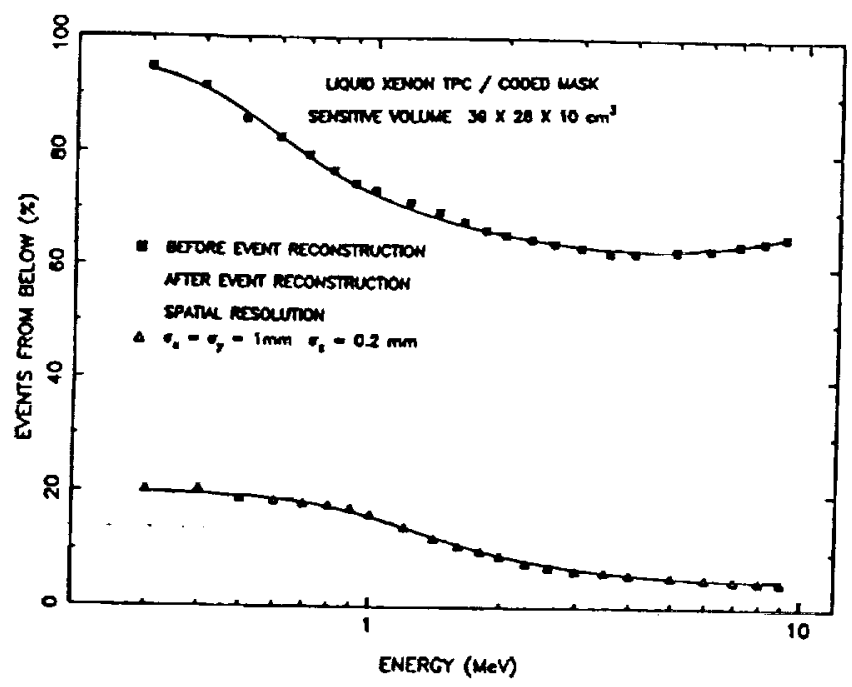

Fig. 6. Percentage of events entering the LXe-TPC from the bottom before and after event reconstruction.

discrimination of the scintillation light pulses offers additional background rejection capability, as it is strongly dependent on the ionization density of the event. For example, neutron induced events can easily be discriminated against, based on the light signature. Figure 7 shows the calculated background flux, before and after applying the Compton event reconstruction of section 2.2 and the fiducial volume cut. We assumed a float altitude of $3 \mathrm{~g} / \mathrm{cm}^{2}$, over Palestine, Texas. The integrated flux over the 0.1-10 $\mathrm{MeV}$ region gives about 340 counts/sec, consistent with typical background rates measured at these altitudes. A background reduction of almost a factor of 10 is obtained by identifying gamma-rays which kinematically couldn't have come through the FOV of the telescope. It is clear that even better background suppression can be achieved,

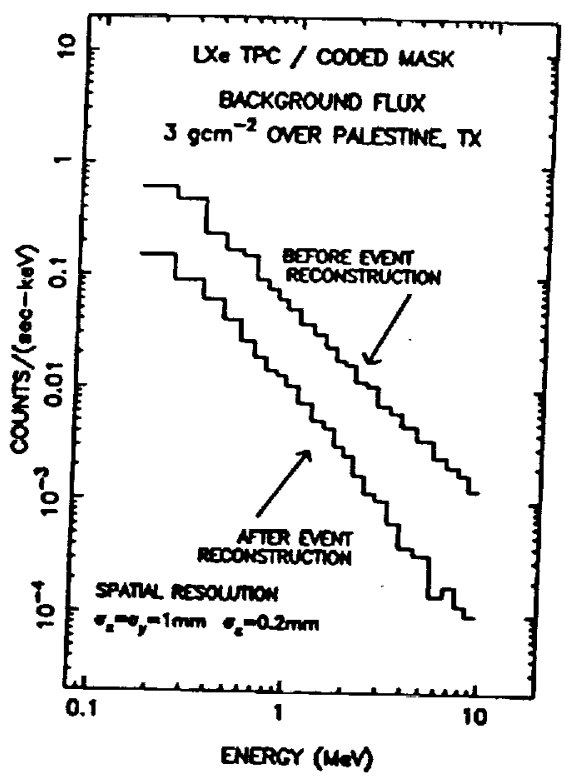

Fig. 7. Calculated background count tate before and after event
reconstruction. 
on an event-by-event basis, using different algorithms for different ganma-ray energies, as previously discussed.

From the calculated efficiency and background rate we have estimated the $3 \sigma$ minimum flux sensitivity of the proposed telescope. The results are shown in Fig. $8 \mathrm{a}$ and 8 b. For a typical exposure of $3 \times 10^{4} \mathrm{~s}$, we find a value of $9 \times 10^{-5}$ photons $/ \mathrm{cm}^{2} \mathrm{sec}$ for $511 \mathrm{keV}$ and of $6 \times 10^{-5}$

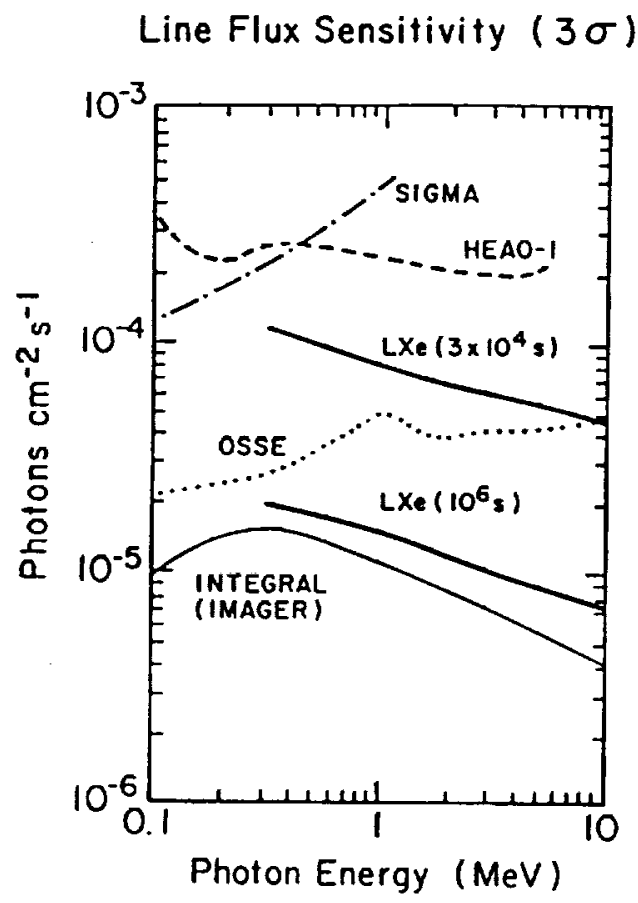

\section{Continuum Flux Sensitivity $(3 \sigma)$}

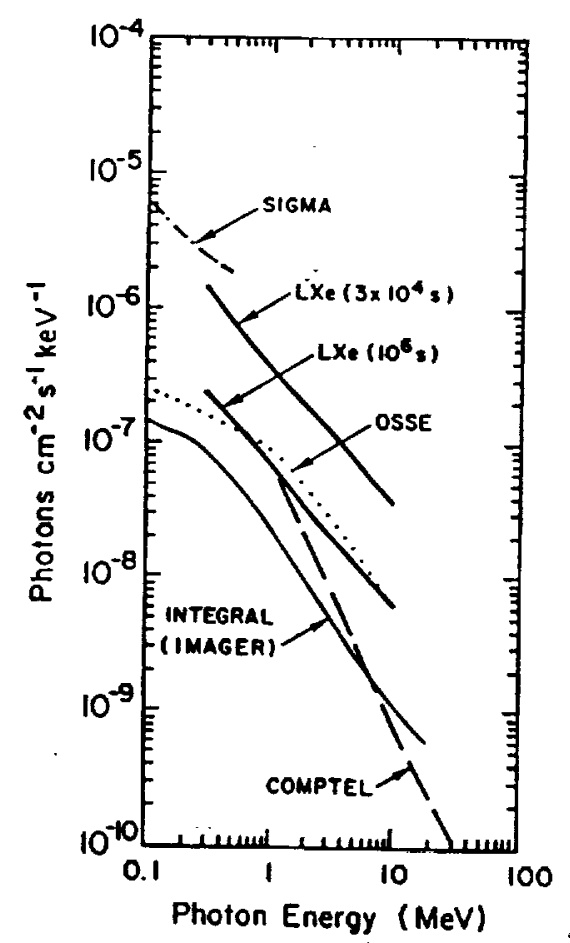

Fic. 8. The $3 \sigma$ minimum (a) line and (b) continuum flux enaitivity of the LXe-TPC/coded mask $\gamma$-ray telescope. photons $/ \mathrm{cm}^{2} \mathrm{sec}$ for $1.8 \mathrm{MeV}$. A continuum sensitivity of $3 \times 10^{-7}$ photons $/ \mathrm{cm}^{2} \mathrm{sec}$ is obtained at $1 \mathrm{MeV}$. The sensitivity curves of the other instruments, shown for comparison, have been taken from Winkler (1991) [12]. When combined with the arcminute source localization accuracy, the high sensitivity of the LXe-TPC telescope makes it competitive with many satellite instruments, even with the much shorter observation time available in a balloon flight.

\section{CONCLUSION}

Monte Carlo modeling of a realistic LXe-TPC/coded mask telescope has demonstrated the unique capability of this instrument to suppress background events as a direct consequence of the imaging properties of the LXe-TPC. By using a reconstruction algorithm for multiple site events, based on the kinematics of Compton scattering, we find that the expected background flux at balloon altitude is reduced by nearly an order of magnitude, resulting in superior line flux sensitivity in the $\mathrm{MeV}$ region, compared to that of exisling scintillator-coded mask or proposed segmented germanium/coded mask $\gamma$-ray telescopes. It is clear that this is only a first step towards the application of the liquid xenon technique for precise imaging of cosmic $\gamma$-ray sources. The full potential will be realized when a detector with large effective area, improved energy resolution and sub-millimeter spatial resolution becomes practically operational as a Compton/pair production $\gamma$-ray telescope.

This work was supported by NASA grant NAGW2013.

\section{REFERENCES}

[1] E. Aprile, R. Mukherjee and M. Suzuki, EUV, X-Ray and Gamma-Ray Instrumentation for Astronomy and Atomic Physics, SPIE Conference Proceedings, ed. C.J. Hailey and O.H.W. Siegmund, 1159 (1989) 295.

[2] P.P. Dunphy et al., Nucl. Instr. and Meth. A274 (1989) 362.

[3] W.E. Althouse et al., Proc. 20th Int. Cosmic Ray Conf. 1 (1987) 84.

[4] J. Paul et al., Cospar Proc. on "Recent result and perspective instrumental developments in $X$ and gammaray astronomy", Adv. Space Res. 11 No. 8 (1991) 289.

[5] E. Aprile et al., Nucl. Instr. and Meth. A316 (1992) 29

[6] Prof. E. Chupp, Univ. of New Hampshire, private communications (1992).

[7] E. Aprile, R. Mukherjee and M. Suzuki, Nucl. Instr. and Meth. A302 (1991) 177.

[8] E.E. Fenimore and T.M. Cannon, Appl. Opt. 17, (1978) 337.

[9] R.L. Ford and W.R. Nelson, The EGS4 Code System, SLAC-210 (1978).

[10] E. Costa et al., Astrophysics and Space Science 100 (1984) 165.

[11] V. Schönfelder, V. Graser and J. Daugherty, Astrophys. J. 217 (1977) 306.

[12] C. Winkler, "Gamma ray line astrophysics", AIP conference proc. 232 (1991) 483. 